\title{
Weltklassepatente - das „Gold“ der Wissensökonomie?
}

Für Volkswirtschaften ist die eigene Innovationsfähigkeit ein entscheidender Faktor, um in der Wissensgesellschaft erfolgreich sein zu können. Das Innovationspotenzial wird häufig mit der Zahl der Patentanmeldungen gemessen - ohne dabei nach Bedeutung zu differenzieren oder ihre tatsächliche Anwendung zu untersuchen. Unser Ansatz versucht dagegen, ein realitätsnäheres Bild zu zeichnen, indem sie nur die wichtigsten Patente (Weltklassepatente) in den Blick nimmt. Die Ergebnisse zeigen, dass der Vorsprung der USA im Patentbereich bei dieser Betrachtungsweise noch deutlicher zutage tritt, China auch in Sachen Patentqualität stark aufholt und Europa vor allem in digitalen Technologien dringenden Handlungsbedarf hat.

\begin{abstract}
Urheberrechte und Innovationsvermögen stehen in der Wissensgesellschaft immer mehr im Zentrum der Debatte um die internationale Wettbewerbs- und Zukunftsfähigkeit von Volkswirtschaften. Im Optimum entsprechen die Kosten für Forschung und Entwicklung dem erwarteten Nutzen durch die Innovation (first best solution). Da dieser Zustand aufgrund von Marktversagen (Anreiz- und Koordinierungsprobleme) in der Praxis faktisch nicht erreichbar ist, greift das Patentsystem von staatlicher Seite ein. Dies wird häufig als „second best solution" bezeichnet (Pénin und Neicu, 2018).
\end{abstract}

Innovationslandschaften werden neben anderen Faktoren also auch stark vom Wettbewerb im Patentsystem

\footnotetext{
(C) Der/die Autor(en) 2020. Open Access: Dieser Artikel wird unter der Creative Commons Namensnennung 4.0 International Lizenz (https:// creativecommons.org/licenses/by/4.0/deed.de) veröffentlicht.
}

Open Access wird durch die ZBW - Leibniz-Informationszentrum Wirtschaft gefördert.

Markus Overdiek, M.Sc., ist Project Manager bei der Bertelsmann Stiftung in Gütersloh.

Thomas Rausch, M.A., ist dort Senior Project Manager.

Kai Gramke, M.A., ist Geschäftsführer der EconSight $\mathrm{GmbH}$ in Basel, Schweiz. geprägt. Ein Patent kommt dabei notwendigerweise aus dem technischen Bereich, und für die Patentfähigkeit müssen beispielsweise in Deutschland folgende drei Kriterien erfüllt sein (Deutsches Patent- und Markenamt, 2019):

- Neuheit: Die Erfindung geht über den aktuellen Stand der Technik hinaus.

- Erfinderische Tätigkeit: Die Erfindung stellt eine signifikante Neuerung dar.

- Gewerbliche Anwendbarkeit: Die Erfindung kann gewerblich genutzt werden.

Patente schaffen einerseits einen Anreiz für Innovationen, weil sie ihren Besitzern ein Verbotsrecht gegenüber Dritten einräumen. Andererseits kann dieses zeitlich befristete Monopol aber auch weiteren Fortschritt verhindern. Die Komplexität des Patentsystems an sich wird oft als Innovationshemmnis genannt. Neue Analysekonzepte und Bewertungssysteme helfen dabei, die Komplexität zu reduzieren und relevante Informationen zu identifizieren. Einige dieser neuen Ansätze haben wir im Rahmen einer Studie, die wir in diesem Artikel vorstellen, angewendet (Bertelsmann Stiftung, 2020b).

\section{Weltklassepatente: Qualität statt Quantität}

Die meisten Untersuchungen zu Patenten haben aus unserer Sicht drei Schwächen, die zu verzerrten Ergebnissen führen:

- Sie betrachten alle Patentanmeldungen, ohne Unterschiede in der Qualität abzubilden. Diese sind jedoch 
beträchtlich: Während nur einige wenige Patente sehr bedeutsam und entsprechend wertvoll sind, ist der Großteil der Patente in puncto Bedeutung und Wert eher vernachlässigbar.

- Sie befassen sich oft mit der Gesamtschau aller Technologien, ohne zu berücksichtigen, dass einige davon (z.B. künstliche Intelligenz) für die Innovationskraft von Volkswirtschaften wesentlich wichtiger sind als andere (z.B. konventionelle Verbrennungsantriebe).

- Sie nehmen nur die Patentanmeldungen in den Blick und vernachlässigen dabei, welche Patente überhaupt genutzt werden bzw. aktiv sind.

Wir versuchen, diese Defizite zu beseitigen und betrachten nur die $10 \%$ der wichtigsten Patente („Weltklassepatente"), konzentrieren uns auf 58 Zukunftstechnologien und analysieren das aktive Patentportfolio von Staaten im Zeitraum von 2000 bis 2019 (Bertelsmann Stiftung, 2020b).

Weltklassepatente werden dabei auf der Grundlage eines Ansatzes von Ernst und Omland (2011) bestimmt. Um die Bedeutung eines Patentes zu ermessen, wird anhand von zwei Kriterien ein „Competitive Impact“ berechnet. Das erste Kriterium ist die Marktabdeckung. Sie ist ein Indikator im Hinblick darauf, als wie bedeutend der Anmelder sein Patent selbst einschätzt. Die Marktdeckung erfasst, in wie vielen Ländern das Schutzrecht angemeldet wird. Dabei findet eine Gewichtung anhand der Größe des Marktes statt. Ein aktives Patent in den USA wird also stärker gewichtet als ein aktives Patent in Deutschland. Das zweite Kriterium ist die technologische Relevanz. Sie spiegelt die Fremdeinschätzung eines Patentes durch die Prüfer in den Patentämtern wider. Diese ziehen zur Prüfung von Patentanmeldungen bereits erteilte Patente heran. Die technologische Relevanz ist generell umso höher, je häufiger ein Patent zitiert wird. Diese Bewertung wird zusätzlich um die Zahl der Jahre korrigiert, die ein Patent bereits aktiv ist, da ältere Patente ansonsten übermäßig gewichtet würden.

Unser Ziel ist es, möglichst passgenaue Aussagen für das Innovationsgeschehen in Technologien zu bestimmen, die in den kommenden Jahren von hoher Relevanz sein werden. Dazu betrachten wir 58 ganz konkrete Zukunftstechnologien wie etwa künstliche Intelligenz, 5G, Functional Food, 3D-Druck oder autonomes Fahren. Diese Technologien haben nach unserer Einschätzung das Potenzial, Wirtschaft und Gesellschaft nachhaltig zu verändern.

In einem weiteren Schritt haben wir die 58 Zukunftstechnologien zehn Technologiebereichen zugeordnet, um große Linien und Entwicklungen besser fassbar zu machen. Diese Technologiebereiche sind: Umwelt, Energie, Ernährung, Infrastruktur, Digitalisierung, Sicherheit, Materialien, Gesundheit, Mobilität und Industrie.

Schließlich richtet sich unser Blick nicht einfach nur auf die Patentanmeldungen, sondern nach dem ReportingDate-Konzept auf das aktive Patentportfolio zum 31.12. eines jeden Jahres im Zeitraum von 2000 bis 2019 (2019: bis zum 9.9.). Durch dieses Vorgehen werden alle noch aktiven Patente aus den Vorjahren mit in die Betrachtung einbezogen, also jene Patente, die noch nicht ausgelaufen sind und für die die Unternehmen durchgehend Gebühren entrichten. Die Daten zu den aktiven Patentportfolios entstammen einer Vielzahl nationaler Patentämter und wurden mithilfe von Big-Data-Analysen zusammengeführt.

Der Blick auf die Technologie Windkraft macht exemplarisch deutlich, wie sich unsere Ergebnisse durch diese fokussierte Betrachtungsweise von den Ergebnissen anderer Analysen unterscheiden. Zum 9. September 2019 gab es weltweit 40.011 aktive Windkraftpatente. Von diesen kamen 16.740 aus China und 3.829 aus Deutschland. Weltklasse sind in der Windkrafttechnologie dennoch lediglich 300 chinesische Patente. Aus Deutschland sind es 958. Die Patenteffizienz in der Windkrafttechnologie beträgt in China also nur gut $2 \%$, in Deutschland dagegen ca. 25 \%. Mit anderen Worten: Ein Blick auf die reinen Anmeldungen aller Patente in der Windkrafttechnologie überzeichnet die chinesische Innovationskraft enorm und unterschätzt die Stärke Deutschlands.

\section{Ostasien: China und Südkorea massiv auf dem Vormarsch}

Da Ostasien, Nordamerika und Europa mit Abstand die höchste Patentaktivität aufweisen, konzentriert sich unsere Studie vor allem auf diese drei Regionen. In Ostasien ist die große Dynamik in China und Südkorea bemerkenswert. Das gerade genannte Beispiel zur Windkrafttechnologie sollte keinesfalls den Eindruck vermitteln, dass China keine Patentqualität zu bieten hat - das Gegenteil ist der Fall: Getrieben von der nationalen Innovationsstrategie „Made in China 2025“, entwickelt sich das Land sukzessive von der „Fabrik der Welt“ zum „Forschungslabor der Welt“ (Jungbluth, 2015; Bertelsmann Stiftung, 2020a). Noch im Jahr 2000 war China kein einziges Mal in einer Technologie unter den fünf Nationen mit den meisten Weltklassepatenten vertreten. 2010 verfügte es in keiner Technologie über einen Platz unter den drei Nationen mit den meisten Weltklassepatenten. Doch im Jahr 2019 rangierte es bereits in 42 der 58 betrachteten Zukunftstechnologien unter den führenden drei Ländern. Besonders stark ist China in den Technologiebereichen Umwelt 


\section{Abbildung 1}

\section{Düngemittel}

Anteile von Weltklassepatenten

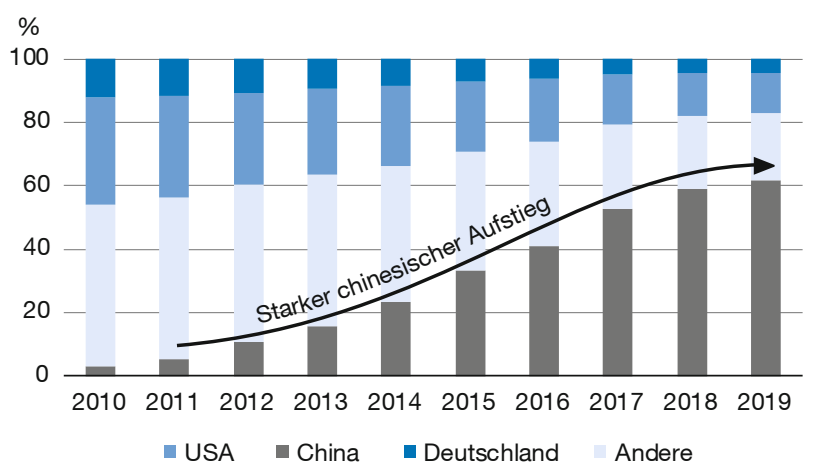

Quelle: Bertelsmann Stiftung, 2020b.

Abbildung 2

5G-Technologie

Zahl von Weltklassepatenten

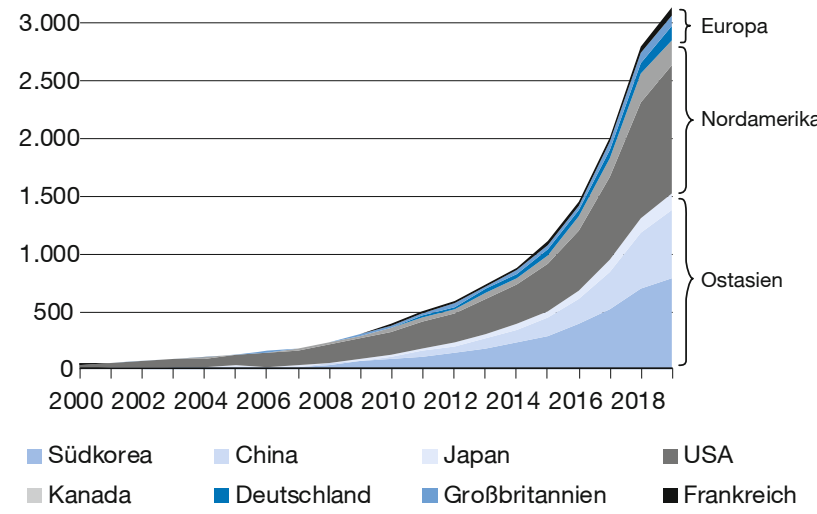

Quelle: Bertelsmann Stiftung, 2020b.

\section{Abbildung 3}

\section{Elektromobilität}

Technologien, in denen Japan im internationalen Vergleich über den höchsten Anteil von Weltklassepatenten verfügt (2019)

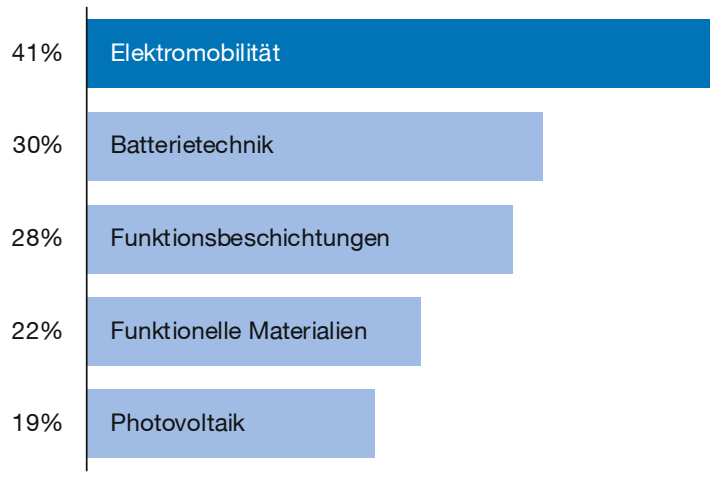

Quelle: Bertelsmann Stiftung, 2020b. und Ernährung. Hier finden sich die fünf Technologien, in denen China das Feld anführt: Recycling, Wasseraufbereitung, Abfallwirtschaft, Biozide und Düngemittel. Am eindrucksvollsten ist die chinesische Dynamik bei den Düngemitteln (vgl. Abbildung 1). Schwächer ist China hingegen im technologisch sehr anspruchsvollen Gesundheitsbereich. Dort befindet es sich nur in einer von sieben Technologien unter den Top Drei.

Eine ähnliche Wachstumsdynamik wie China, wenn auch in einem kleineren Maßstab, weist Südkorea auf. Hier sei beispielhaft die Technologie 5G genannt, in der sich vor allem der größte südkoreanische Konzern Samsung besonders stark etabliert hat. Bei der 5G-Technologie weist Südkorea mit 792 Weltklassepatenten einen für seine Größe außerordentlich beachtlichen Anteil von $19 \%$ aller Weltklassepatente auf (vgl. Abbildung 2). Zu dieser Stärke passen die hohen Wachstumsraten bei Weltklassepatenten in digitalen Technologien aus dem Bereich künstliche Intelligenz, Big Data oder Cloud Computing. Insgesamt hat sich Südkorea 2019 bereits in der Hälfte der 58 untersuchten Technologien einen Platz unter den fünf Nationen mit den meisten Weltklassepatenten gesichert.

Japan verzeichnet dagegen - anders als China und Südkorea - einen langsamen Abstieg auf hohem Niveau. Im Jahr 2000 befand sich Japan noch in 49 Technologien unter den drei Ländern mit den meisten Weltklassepatenten, 2019 sind es nur noch 38. Als stärkste Technologie Japans ist ganz klar die Elektromobilität hervorzuheben. Hier ist Japans Anteil an Weltklassepatenten im internationalen Vergleich mit $41 \%$ am höchsten (vgl. Abbildung 3).

\section{Nordamerika: USA immer noch klarer Spitzenreiter}

Die USA dominieren das weltweite Geschehen bei den besonders wichtigen Patenten. Sie weisen, unter anderem dank gezielter staatlicher Förderprogramme (z.B. der Defense Advanced Research Projects Agency [DARPA], in 50 der 58 betrachteten Zukunftstechnologien die meisten Weltklassepatente auf und befinden sich in den verbleibenden acht Technologien auf dem zweiten Rang. Diese Werte sollen jedoch nicht darüber hinwegtäuschen, dass die USA in manchen Technologiebereichen wie Ernährung oder Umwelt massiv Anteile abgeben mussten - vor allem an China. Deutlich führend sind die USA aber immer noch in den Bereichen Sicherheit und Digitalisierung. Dort spüren sie zwar ebenso die aufkeimende Konkurrenz aus anderen Nationen, können jedoch noch mit gut der Hälfte aller Weltklassepatente aufwarten (vgl. Abbildung 4).

Kanada kann aufgrund seiner geringen Einwohnerzahl und wesentlich kleineren Volkswirtschaft kaum so hohe 
Abbildung 4

\section{Digitalisierung}

Anteile von Weltklassepatenten

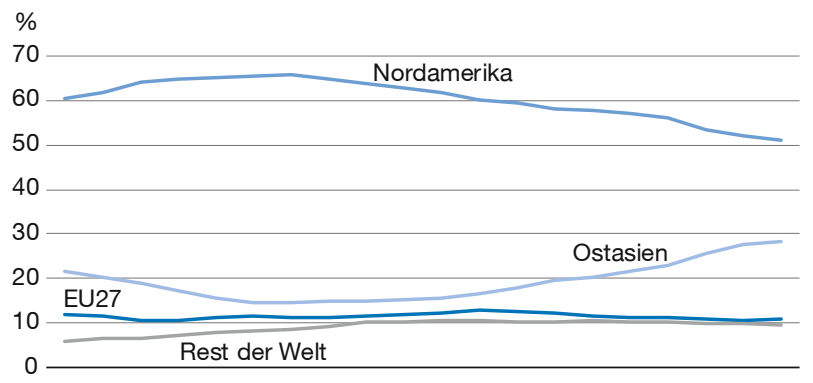

$2000200220042006200820102012 \quad 2014 \quad 2016 \quad 2018$

Quelle: Bertelsmann Stiftung, 2020b.

\section{Abbildung 5}

Quantencomputing

Weltklassepatente je 1 Mio. Einwohner (2019)

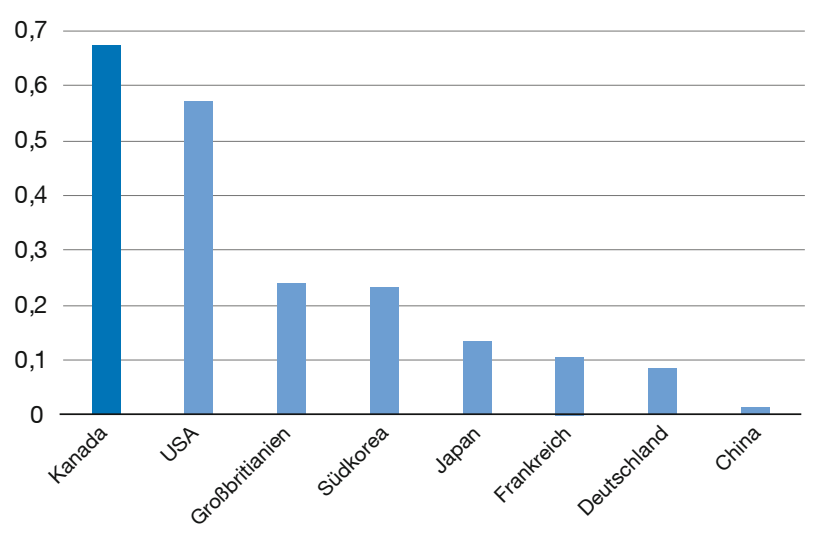

Quelle: Bertelsmann Stiftung, 2020b.

\section{Abbildung 6}

3D-Druck

Anteile von Weltklassepatenten

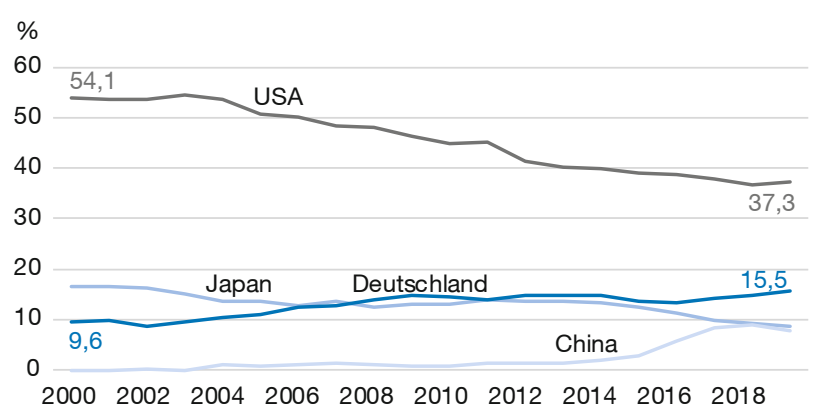

Quelle: Bertelsmann Stiftung, 2020b. absolute Weltklassepatentzahlen verbuchen wie die USA. In Relation zu seiner Bevölkerungsstärke ist Kanada aber in einzelnen Bereichen, insbesondere bei der Digitalisierung, sehr stark. So weist Kanada z. B. die meisten Weltklassepatente pro 1 Million Einwohner in der Technologie Quantencomputing auf (vgl. Abbildung 5).

\section{Europa: Gemeinsam oft stark, aber nicht bei der Digitalisierung}

Deutschland ist die stärkste europäische Forschungsnation. Daran hat sich in den letzten 20 Jahren wenig geändert. Genau wie Europa insgesamt hat es jedoch in einigen Zukunftstechnologien Anteile an Weltklassepatenten eingebüßt. Hervorzuheben ist die deutsche Stärke in den Gesundheitstechnologien. Während Deutschland in keiner Zukunftstechnologie den ersten Platz im internationalen Weltklassepatentranking erreicht, liegt es siebenmal auf dem zweiten Platz - viermal davon im Bereich Gesundheit. Mit dabei ist auch die im Kontext der Corona-Krise bedeutende Technologie der Impfstoffe. In Bereichen, die als seine traditionellen Stärken gelten wie Industrie oder Mobilität -, ist Deutschland zwar noch gut aufgestellt, gleichzeitig aber auch zunehmend unter Druck. So hat Deutschland in den letzten beiden Dekaden Anteile in Technologien wie dem autonomen Fahren oder der Prozessautomatisierung verloren. Jedoch konnte es, wie das Beispiel 3D-Druck zeigt, partiell auch Anteile hinzugewinnen (vgl. Abbildung 6).

Besondere Schwäche zeigt Deutschland hingegen in Technologien aus dem Bereich Digitalisierung. Da diese Technologien zunehmend für die Innovationsfähigkeit in anderen Bereichen entscheidend sind, könnte sich dieses Defizit auch dort mittel- und langfristig negativ auswirken.

Frankreichs stärkste Technologie ist mit Abstand die Luftund Raumfahrt (vgl. Abbildung 7). Dieses Ergebnis ist für die Nation mit einer langen Raumfahrttradition und dem Hauptsitz von Airbus nicht überraschend. Fraglich ist jedoch, welche Spuren die Corona-Krise in dieser Technologie hinterlassen wird. Möglicherweise setzt sich dadurch der starke Abstieg Frankreichs weiter fort: Im Jahr 2000 war es gemessen an den Weltklassepatenten noch in 41 von 58 Technologien unter den besten fünf Nationen, 2019 ist dieser Wert auf zwölf gesunken.

Der Brexit könnte auch für die europäische Innovationslandschaft nachhaltige Konsequenzen haben. Aus EUPerspektive stellt insbesondere die gute Performance Großbritanniens in den Bereichen Gesundheit und - für europäische Verhältnisse außergewöhnlich - Digitalisierung einen herben Verlust dar. Hinzu kommen noch ver- 


\section{Abbildung 7}

\section{Luft- und Raumfahrt}

Technologien, in denen Frankreich im internationalen Vergleich über den höchsten Anteil von Weltklassepatenten verfügt (2019)

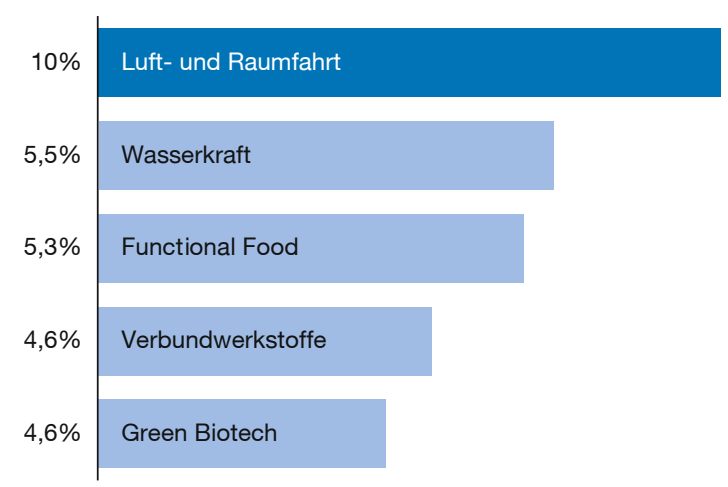

Quelle: Bertelsmann Stiftung, 2020b.

einzelte technologische Stärken in anderen Bereichen, wie etwa in der Wasserkraft (vgl. Abbildung 8).

Europa ist insgesamt, gemessen an seinem Output an Weltklassepatenten in Zukunftstechnologien, eine sehr heterogene Forschungslandschaft. Normiert auf die Patente pro 1 Million Einwohner ist die Schweiz deutlich führend. In Süd- und Osteuropa ist die Forschungsaktivität hingegen deutlich geringer als in Nord- und Westeuropa (vgl. Abbildung 9).

\section{Abbildung 9}

\section{Forschungsaktivität in Europa}

Forschungsaktivität gemessen als Weltklassepatente je 1 Mio. Einwohner (2019)

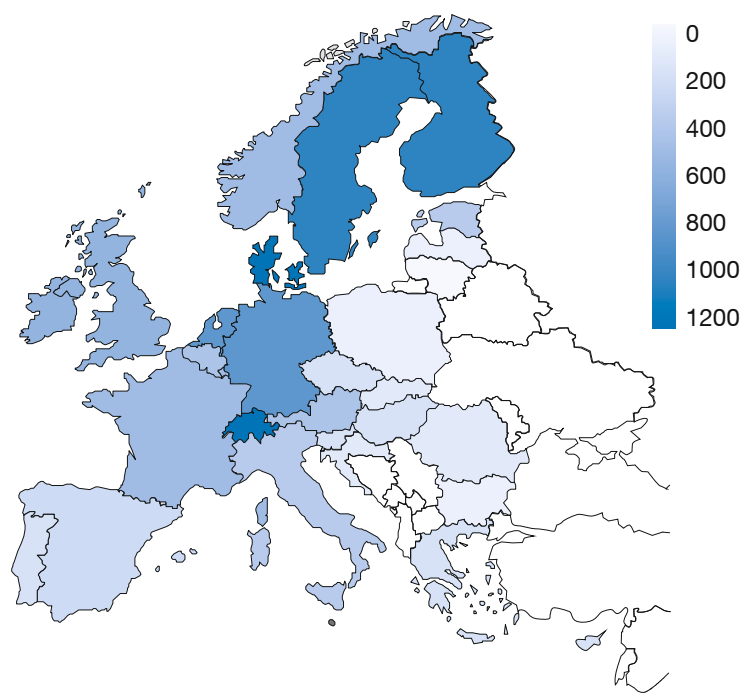

Quelle: Bertelsmann Stiftung, 2020b.

\section{Abbildung 8}

Wasserkraft

Platzierungen der Staaten innerhalb Europas mit den meisten Weltklassepatenten

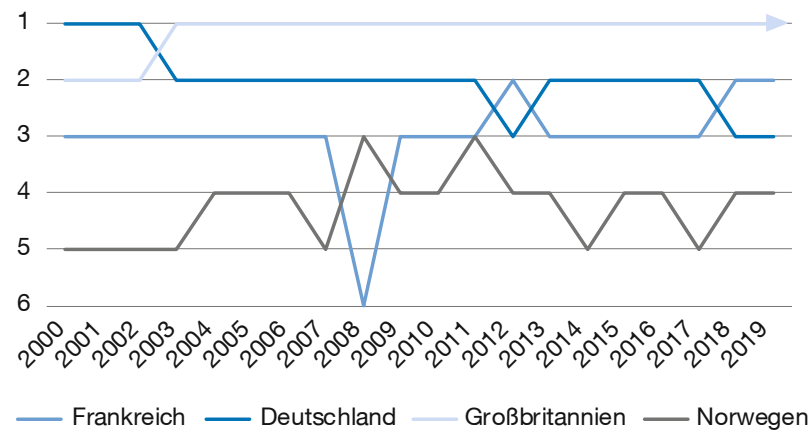

Quelle: Bertelsmann Stiftung, 2020b.

\section{Schlussfolgerungen}

Aus den Ergebnissen ergeben sich für uns drei Vorschläge zur Stärkung der Innovationslandschaft in Deutschland und Europa:

1. Europäische und internationale Kooperationen ausbauen: EU-Staaten sind in den Zukunftstechnologien besonders gut aufgestellt, in denen sie mit anderen europäischen Staaten kooperieren (vgl. Abbildung 10). Als Einheit kann Europa also mit anderen Weltregionen mithalten. Diese europäischen Forschungskooperationen bringen im Idealfall die guten Eigenschaften von Patenten mit dem Open-Innovation-Ansatz überein, sodass die Gesellschaft als Ganzes profitieren kann. Auch staatliche Institutionen können bei internationalen Kooperationen verstärkt eine tragende Rolle spie-

\section{Abbildung 10}

\section{Forschungskooperationen in der EU}

Anteil der Weltklassepatente mit Forschern aus mindestens zwei EUStaaten (2019) in \%

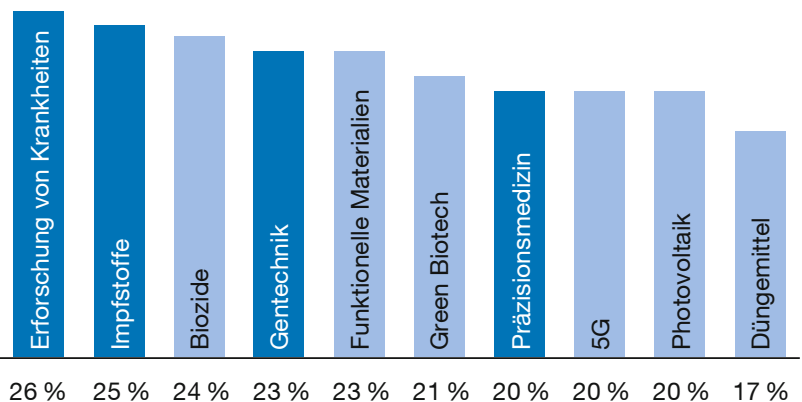

Quelle: Bertelsmann Stiftung, 2020b. 
len. In anderen Weltregionen sind diese Akteure ohnehin ein zentraler Bestandteil der Innovationspolitik (Bertelsmann Stiftung, 2020a).

2. Bessere Anwendung und Kommerzialisierung: Deutschland und Europa verfügen noch immer über herausragende Forschungsstandorte. Problematisch ist jedoch zumeist der Transfer in die Praxis. Der Schritt in Anwendung und Kommerzialisierung kann noch verbessert und die Förderung von Gründungswilligen sollte gestärkt werden. Wichtig ist in diesem Zuge auch eine bessere Vernetzung von der angewandten Forschung und Entwicklung auf Unternehmensseite mit der Grundlagenforschung an Universitäten und Instituten. Hier sehen sich beide Seiten noch zu oft in gegenseitiger Bringschuld - mit dem Ergebnis, dass es häufig zu keinen gemeinsamen Aktivitäten kommt.

\section{Innovation und gesellschaftlichen Fortschritt verbinden:} Innovationspolitik sollte sich nicht nur wirtschaftlich, sondern in gleichem Maße auch gesellschaftlich ambitionierte Ziele setzen (Mazzucato, 2014). Aufgrund der Corona-Krise hat die Frage nach der Gestaltung einer sozialökologischen Transformation von Wirtschaft und Gesellschaft noch einmal an Relevanz gewonnen. In welche Richtung Innovationspolitik und damit auch Fortschritt konkret gehen sollen, ist dabei notwendigerweise Teil eines gesellschaftlichen Aushandlungsprozesses. Argumente müssen hierfür offen ausgetauscht werden, sodass allen Beteiligten mögliche Konsequenzen bestimmter Richtungsentscheidungen bewusst sind.

\section{Literatur}

Bertelsmann Stiftung (2020a), Von Trump und Xi lernen? Globalisierung und Innovation als Treiber einer neuen Industriepolitik, GED Focus Paper.

Bertelsmann Stiftung (2020b), Weltklassepatente in Zukunftstechnologien. Die Innovationskraft Ostasiens, Nordamerikas und Europas.

Deutsches Patent- und Markenamt (2018), Patente, https://www.dpma. de/patente/patentschutz/schutzvoraussetzungen/index.html (17. Juli 2020).

Ernst, H. und N. Omland (2011), The Patent Asset Index - A New Approach to Benchmark Patent Portfolio, World Patent Information, 33(1), 34-41.

Jungbluth, C. (2015), Going Global - Die internationale Expansion chinesischer Unternehmen, Nomos.

Mazzucato, M. (2014), Das Kapital des Staates. Eine andere Geschichte von Innovation und Wachstum, Kunstmann.

Pénin, J. und D. Neicu (2018), Patents and Open Innovation: Bad Fences Do Not Make Good Neighbors, Journal of Innovation Economics \& Management, 25(1), 57-85.

Title: World Class Patents - The Gold of the Knowledge Economy?

Abstract: Innovation is the foundation of social progress and international competitiveness. Based on a new approach to patent assessment, we show how the innovative power of major economies and world regions has shifted between 2000 and 2019 . Analysing the top ten percent of the most important patents from the active patent portfolio in 58 cutting-edge technologies (world class patents), we find that the international balance of power in innovation is shifting in favour of East Asia. While the United States still enjoys a sizeable lead in many technologies, China is catching up fast, particularly in environment and nutrition. Europe has trouble keeping the pace, particulary in digitalisation.

JEL Classification: 030, О32, О33. 